\title{
TEMAS Y PROBLEMAS DE LA HISTORIA POLÍTICA ARGENTINA (1890-1916)*
}

\author{
Carlos Malamud*
}

El período que va desde la "Revolución del Parque", en 1890, y que marcó el surgimiento de la Unión Cívica (UC), hasta la llegada de los radicales al poder de la mano de Hipólito Yrigoyen, en 1916, es, desde el punto de vista de la Historia Política, una época de grandes transformaciones y que últimamente está recibiendo una nueva y creciente atención. Por eso, en estos momentos, coincidiendo con una indudable recuperación de la disciplina, vale la pena preguntarnos dónde estamos, cuáles son los principales problemas que atraviesa la investigación, cuáles son las líneas abiertas y cuáles las preguntas en las que más se debería insistir. Sin ánimos de ser exhaustivo, recorreré un camino previamente transitado (1), centrándome básicamente en la producción historiográfica de los últimos años.

La obra pionera de Natalio Botana (2) tuvo la virtud de insistir en el estudio de los principales mecanismos del "orden conservador", de la mano de Hipólito Yrigoyen en 1916, incorporando a la Historia Política argentina categorías y problemas propios de la Ciencia Política. Hasta mediados de la década de los 70 los escasos intentos de traspasar los lineamientos tradicionales de la Historia Politica habían tenido bastante poco éxito, especialmente por el predominio que mostraban la Historia Económica y la Historia Social, bajo la influencia de la Escuela de Annales, del marxismo y de la revolución (¿social?, ¿antiimperialista?, ¿nacional y popular?), que entonces muchos veían inminente. En ese momento, sólo algunos trabajos de Historia de las Ideas aportaban algo de frescura a un medio académico e intelectual poco interesado en una Historia Política dominada por lo fáctico y lo institucional (3). Inclusive este punto era tratado con ciertas reservas, y algunos temas apenas han

* Se presenta aqui la versión inicial de un trabajo en marcha sobre la Historia Política argentina del periodo 1890-1946.

** UNED-I.U. Ortega y Gasset, Madrid.

1. Ver, fundamentalmente, Ezequiel Gallo: "Historiografia política: 1880-1900", Comité Internacional de Ciencias Históricas (Comité Argentino), Historiografia Argentina (1858-1988). Una evaluación critica de la producción histórica argentina, Bs. As., 1990

2. Natalio Botana: El orden conservador. La politica argentina entre 1880 y 1916, Ed. Sudamericana, Bs. As., 1977. En ella se inspiran numerosos trabajos, como el de Flavio Fiorani, La fine del caudillismo. Politica e istituzioni liberali in Argentina (1880-1916), Edizioni Associate, Roma, 1990. En Gustavo Ferrari y Ezequiel GaIlo (eds.), La Argentina del Ochenta al Centenario, Ed. Sudamericana, Bs. As., 1980, se encuentra bastante bien representado el estado de cosas existente a finales de la década del 70.

3. Un enfoque innovador es el de Mario Serrafero, "El presidencialismo en el sistema politico argentino", tesis doctoral, Instituto Universitario Ortega y Gasset (U. Complutense), 1992. avanzado, como aquellos vinculados a la Historia del Poder Judicial, o a conceptos como "ley y orden", etc.

Según Botana, el orden conservador se asentaba en el dominio hegemónico del Partido Autonomista Nacional (PAN) y en la alianza tejida entre el presidente de la $\mathrm{Na}$ ción, el gran elector del sistema, y los gobernadores. La necesidad que el primer mandatario tenía de los gobernadores y el respaldo que requerían estos últimos del poder presidencial, de un poder presidencial pleno de resortes, como la intervención federal, garantizaban el funcionamiento de un orden algo inestable y que para mantenerse debía explotar a tope la "máquina política" gubernamental (4).

Estamos frente a un sistema que, salvo en la provincia de Buenos Aires y la Capital Federal, dejaba poco margen de maniobra para los partidos de oposición. Si bien en las elecciones legislativas no se votaba por listas cerradas, y los candidatos más votados hasta cubrir el número de vacantes resultaban electos, se trataba de algo bastante parecido al "winner takes all", aunque no era exactamente eso, como se suele afirmar corrientemente. Botana también planteó algunas cuestiones que merecerían mayor atención, como la importancia de lo provincial y local en la vida política argentina (5).

De todos modos es bastante poco lo que hoy sabemos del PAN (6), un tema que requiere mayor investigación empírica. Si bien se insiste con cierta intensidad en los conflictos que enfrentaron a Roca y Carlos Pellegrini, se deberían tener más en cuenta las fuertes contradicciones

4. Dolores Cullen, "Electoral Participation in Argentina at the Turn of the XXth. Century", paper presentado al XIX Congreso de LASA, Washington, 1995, mimeo. En él, Cullen presenta algunas de las cuestiones abordadas más detalladamente en su tesis doctoral, "Electoral Practices in Argentina, 1898-1904", presentada en 1994 en la Universidad de Oxford.

5. En los últimos años mucho se ha avanzado al respecto, aunque todavia hay numerosos interrogantes. Del tema me ocupo en el capitulo III, "La cuestión municipal en la vida política argentina en torno al Centenario", de mi Partidos politicos y elecciones en Argentina: La Liga del Sur Santafesina (1908-1916), (en prensa). Los siguientes trabajos cubren algunas de las cuestiones vinculadas al problema: Richard Walter: La provincia de Buenos Aires en la politica argentina, 1912-1943. Emecé, Bs. As., 1987 y Politics and Urban Growth in Buenos Aires: 1910-1942, Cambridg University Press, Cambridge, 1993 (un buen estudio de politica municipal de la ciudad de Buenos Aires); Marcela Ternavasio ha realizado una excelente tesis de maestría: "Municipio y política: un vínculo conflictivo. Análisis histórico de la constitución de los espacios locales en Argentina (1850-1920)", Facultad Latinoamericana de Ciencias Sociales, Rosario, 1991. Ver también George Heaps-Nelson: "Argentine Provincial Politics in an Era of Expanding Electoral Participation: Buenos Aires and Mendoza, 1906-1918”, Ph.D. Thesis, University of Florida, 1975 y el más completo trabajo de Donald Peck: "Argentinian Politics and the Province of Mendoza, 1890-1916", Ph. D. Thesis, Oxford University, 1977.

6. Un tema que actualmente está trabajando Paula Alonso. 
entre roquistas y juaristas, pese a la pretensión generalizada de que el gobierno de Juárez Celman fue sólo la línea del continuismo con el primer gobierno de Roca. Sin embargo, Tim Duncan ha presentado una visión más matizada del juarismo y también del proceso que desembocó en la "Revolución del Parque". En este punto pone especial énfasis en las fuerzas políticas porteñas que convergieron en la creación de la UC (7). Pese a ello, pocos han sido los que han seguido avanzando por el fértil territorio desbrozado por Duncan (8).

Nuevas líneas de investigación se dedican a estudiar la naturaleza de las elecciones y los límites de la participación ciudadana (9). Si bien en el sistema electoral argentino no existían restricciones en base a la fortuna o a la condición del elector (salvo en lo atinente a elecciones provinciales y locales, que se regían por una amplia casuística), las mujeres y los extranjeros no participaban en los comicios nacionales. Sin embargo, los últimos podían votar en determinados casos, y lo normal era que la participación de estos grupos en la vida política local no se limitara al contacto, o no, con las urnas (10).

Por lo general, el sistema político argentino habia sido analizado teniendo en cuenta casi exclusivamente la literatura existente, lo que llevaba a aquellos autores que incursionaban en el tema a repetir una y otra vez las preguntas anteriormente formuladas, desde las mismas perspectivas y casi con los mismos prejuicios que en el pasado. Estos defectos se ampliaban cuando el interés pasaba por comprobar teorías de tipo general, abarcadoras del sistema como totalidad (11). Esto explica que algunos temas, como el papel jugado por la pren-

7. William T. Duncan: "Government by Audacity. Politics and the Argentine Economy, 1885-1892", Ph. D. Thesis, University of Melbourne, 1981

8. Una de las pocas excepciones es la tesis doctoral de Paula Alonso: "The Origin of the Argentine Radical Party, 1889-1898", Universidad de Oxford, 1992. De la misma autora ver también: "Politics and Elections in Buenos Aires, 1890-1898": The Perfomance of the Radical Party", Journal of Latin American Studies, Vol. 25, Part 3 (1993).

9. Hilda Sabato: "Participación politica y espacio público en Buenos Aires, 1860-1880: algunas hipótesis", El reformismo en contrapunto, CLAEH, Montevideo, 1989 y "Citizenship, Political Participation and the Formation of the Public Sphere in Buenos Aires 1850s-1880s", Past and Present, N.136 (1992). Ver también sus trabajos con Ema Ciboti: "Hacer politica en Buenos Aires: los italianos en la escena pública porteña, 1860-1880", Boletín del Instituto de Historia Argentina y Americana, N."2 (1990) y con Elias Palti: "¿Quién votaba en Buenos Aires?: Práctica y teoria del sufragio, 1850-1880", Desarrollo Económico, Vol. 30, ‥119 (1990). También Anibal Viguera, "Participación electoral y prácticas politicas en Buenos Aires, 1912-1922", Entrepasados, N.1 1 (1991)

10. Ver los trabajos pioneros de E. Gallo: "Conflictos socio-politicos en las colonias agricolas de Santa Fe (1870-1880)", Documento de Trabajo, Instituto Torcuato Di Tella, Centro de Investigaciones Sociales, N.․ 87, Bs. As., 1973; "Boom cerealero y cambios en la estructura socio-política de Santa Fe (1870-1895)", Documento de Trabajo, Instituto Torcuato Di Tella, Centro de Investigaciones Sociales, N. ${ }^{\circ} 88$, Bs As., 1974 y Colonos en armas. Las revoluciones radicales en la provincia de Santa $\mathrm{Fe}$ (1893). Instituto Torcuato di Tella, Bs. As., 1977. Algunas cuestiones vinculadas a estos puntos son tratadas en: Maristella Svampa, "Inmigración y nacionalidad: el caso de la Argentina, 1880-1910", Studi Emigrazione/Etudes Migrations, Vol. XXX N. 110 (1993); Marta Bonaudo, Silvia Cragnolino y Elida Sonzogni, "La cuestión de la identidad política de los colonos santafesinos: 1880-1898. Estudio de algunas experiencias", Anuario, N. 14 (Escuela de Historia, Rosario, 1991) y "Discusión en torno a la participación politica de los colonos santafesino: Esperanza y San Carlos (1856-1883)", Estudios Migratorios Latinoamericanos, N. 9 (1988); Lilia Ana Bertoni: "La naturalización de los extranjeros, 1887-1893: ¿derechos políticos o nacionalidad?", Desarrollo Económico, Vol. 32, N. 125 (1992); Carina Frid de Silberstein, "Parenti, negozianti e dirigenti: la prima dirigenza italiana di Rosario (1860-1890)", en Giangusto Roseli (ed.), Identita degli italiani in Argentina. Ret sociali/Famiglia/Lavoro, Roma, 1993; Ema Ciboti, "La elite italiana de Buenos Aires: el proyecto de nacionalización del 90", Amuario, N.14 (1991) y Romolo Gandolfo, "Inmigrantes y politica en Argentina: La revolución de 1890 y la campaña en favor de la naturalización automática de residentes extranjeros", Estudios Migratorios Latinoamericanos, N. 17 (1991)

11. Un ejemplo podria ser el libro de Karen Remmer: Party Competition in Argentina and Chile. Political Recruitment and Public Policy, 1890-1930, University of Nebraska Press, 1984, aunque se trata de una obra valiosa por su enfoque comparativo. sa en el sistema político haya sido apenas trabajado (12), mientras que otros, al amparo de las modas académicas, como es el caso de la "construcción de la Nación" o el desarrollo de los "imaginarios colectivos", reciban mayor atención (13).

La reforma electoral, inicialmente planteada por el general Julio A. Roca y su ministro del interior Joaquín V. González, en 1902, y finalmente por el presidente Sáenz Peña e Indalecio Gómez, diez años más tarde, ha merecido la atención de los investigadores en los últimos tiempos (14). Por lo general, las preguntas relacionadas con la reforma tienen que ver con la forma en que ésta permitió la expansión del voto, pero suelen dejar de lado otras implicaciones de la misma, como sus influencias internacionales, el influjo que tuvo en el sistema político nacional, los mecanismos de transmisión de sus principales postulados a las constituciones y las leyes provinciales y la forma en que afectó la competitividad en las elecciones locales y provinciales.

El tema de la reforma electoral está estrechamente relacionado con los orígenes del radicalismo. David Rock (15) recogió los principales tópicos de la tradición yrigoyenista, que en un halo de misterio situaba al radicalismo originario entre la conspiración revolucionaria (ahí están las intentonas de 1890,1893 y 1905) (16) y la abstención. Sin embargo, como bien ha probado Paula Alonso (17), hasta 1898, los radicales solían acudir a las urnas con cierta asiduidad, obteniendo algunos éxitos en la Capital Federal y la provincia de Buenos Aires. Pese a todo, y al igual que en el caso del PAN, todavía sabemos bastante poco del desarrollo del radicalismo en las provincias del interior (18).

Más atención han merecido otras cuestiones algo menores, como el reiterado tema de la influencia del krausismo en el yrigoyenismo (19), aunque lo importante del caso es el impacto del primer republicanismo español en el desarrollo del ideario radical. Paradójicamente, a estas cuestiones, como en general al peso de la política y los políticos españoles en Argentina, y viceversa, no se le ha dado toda la atención que merecen (20).

El estudio de otros partidos políticos no ha merecido suficiente atención en los últimos tiempos, más allá de los trabajos tradicionales sobre el socialismo y el anarquis-

12. Ricardo Sidicaro, La politica mirada desde arriba. Las ideas del diario La Nación, 1909-1989. Ed. Sudamericana, Bs. As., 1993

13. Es el caso del discutible, y discutido, libro de Nicolas Shumway, The Invention of Argentina, University of California Press, 1991 (hay edición en español).

14. Fernando Devoto y Marcela Ferrari (eds.): La Construcción de las democracias rioplatenses: proyectos institucionales y prácticas politicas, 1900-1930, Bs. As., 1994.

15. David Rock: "Machine Politics in Buenos Aires and the Argentine Radical Party, 1912-1930", Journal of Latin American Studies, Vol. 4, N.․ 2 y especialmente El radicalismo argentino, 1890-1930, Ed. Amorrortu, Bs. As., 1977.

16. Roberto Etchepareborda: Tres revoluciones, 1890, 1893, 1905, Editorial Pleamar, Bs. As., 1968

17. Ver n. 9 .

18. E. Gallo y Silvia Sigal: "La formación de los partidos politicos contemporáneos: la UCR (1890-1916)", en Torcuato di Tella, Gino Germani, Jorge Graciarena y colaboradores, Argentina, sociedad de masas, Eudeba, Bs. As., 1966.

19. Horacio Biagini (comp.): Origenes de la democracia argentina. El transfondo krausista, Ed. Legasa, Bs. As., 1989; Osvaldo Alvarez Guerrero: Politica y ética social. Yrigoyen y el krausismo, origenes ideológicos de la UCR. Ed. de la Patagonia, Fuerte General Roca, 1984.

20. La tesis doctoral de Daniel Rivadulla Barrientos: "Relaciones HispanoArgentinas durante el reinado de Alfonso XIII en una perspectiva Atlántica", U. Complutense (1991) refleja, en cierta medida, el ambiente fluido que existia entre ambos paises. Ver también su La "Amistad irreconciliable". España y Argentina. 1900-1914, Ed. Mapfre, Madrid, 1992. José Alvarez Junco, en El emperador del Paralelo, Alianza Editorial, Madrid, 1990, recoge algunas cuestiones importantes de la visita de Lerroux a la Argentina. 
mo (21). En el caso del centro derecha es como si el PAN eclipsara todo el panorama y las restantes opciones existentes, especialmente las que se conformaron a partir de la disolución del PAN, no merecieran gran atención (22). Por otra parte, es bastante corriente que al referirse a la derecha argentina, buena parte de los autores no hablen de conservadores, o incluso de liberales, sino sólo de nacionalistas y fascistas, es decir, del pensamiento autoritario (23). Desde una óptica totalmente diferente Eduardo Zimmerman analiza el componente reformista y social del liberalismo argentino, un tema bastante descuidado hasta la fecha (24).
Estrechamente relacionada con la Historia Política está el género biográfico, un género que no ha tenido importantes cultores en la Historia argentina reciente. Dentro del mismo encontramos ejemplos muy disimiles. Mientras el excelente relato casi novelado de la vida de Roca, de Félix Luna (25), ha sido un éxito de ventas, la biografía de Julio A. Roca (h.), peca de gran superficialidad (26). Como se ha podido ver en esta rápida y somera relación, si bien se ha avanzado mucho en los últimos años, todavía es mucho lo que queda por hacer y son numerosos los temas que abordar con mayor profundidad.

\section{RESUMEN}

Carlos Malamud señala en su artículo los principales problemas con los que se ha encontrado la historia política argentina en el período 1890-1916. Menciona también los mecanismos del "orden conservador" - la vida política local y provincial, el funcionamiento del PAN, etc. - y la importancia de los procesos electorales y participación ciudadana en ese período.

ABSTRACT
Carlos Malamud points out in his article the mean problems of the Argentinian political history in the
period 1890-1916. He mentiones the mechanisms of the "conservative order" - such as the local and
provincial political life, the functioning of PAN, ... - and the importance of the electoral processes and
citizen participation in this period.

21. En Anibal Iturrieta (ed.), El pensamiento politico argentino contemporáneo, Sintesis y Grupo Editor Latinoamericano, Bs. As., 1994, algunos articulos presentan sintesis y perspectivas interesantes. Jeremy Adelman: "Socialism and Democracy in Argentina in the Age of the Second International", HAHR, Vol 72, N.² (1992)

22. Iván Llamazares Valduvieco realiza un interesante análisis comparativo entre el conservadurismo argentino de las primeras décadas del siglo XX con el actual, "Periferias conservadoras. Un análisis comparativo de la evolución del conservadurismo argentino", tesis doctoral, Instituto Universitario Ortega y Gasset (U. Complutense), 1994. Menos éxito han tenido los partidos provinciales. Analizo el caso de la Liga del Sur en "Los partidos políticos en la Argentina, 1890-1914: programas y plataformas. El caso de la Liga del Sur", en Eduardo Posada-Carbó (ed.), Wars, Parties and Nationalism: Essays on the Politics and Society of Nineteenth-Century Latin America, Nineteenth Century Latin America, N.. 1, Institute of Latin American Studies, Londres, 1995 y en la obra citada en n. 6. También tratan de Santa Fé, Marta Bonaudo y Elida Sonzogni, "Redes parentales y facciones en la política santafesina, 1850-1900", Siglo XIX, Revista de Historia. Segunda época, N. 11 (1992). En la obra editada por F. Devoto y M. Ferrari, citada en $\mathrm{n}$. 15, se analizan algunos casos de las provincias de Córdoba y Buenos Aires.

23. Sandra McGee Deutsch y Ronald Dolkart (eds.), The Argentine Right. Its History and Intellectual Origins, 1910 to the Present, SR Books, Wilmington, 1993 y Sandra McGee Deutsch, Counterrevolution in Argentina, 1900-1932. The Argentine Patriotic League, Lincoln, 1986. Doy una visión contraria en "El Partido Demócrata Progresista: un intento fallido de construir un partido nacional liberal-conservador", Desarrollo Económico, Vol. 35, N.²138 (1995). Hasta hace poco tiempo trabajos como el de Oscar Cornblit: "La opción conservadora en la politica argentina", Desarrollo Económico, N.56, Vol. 14 (1975), no eran frecuentes.

24. Eduardo Zimmerman, Los liberales reformistas. La cuestión social en la Argentina, Ed. Sudamericana-Universidad de San Andrés, Bs. As., 1995. Ver también de Tulio Halperin Donghi: "Argentina: Liberalism in a Country Born Liberal", en Joseph Love y Nils Jacobsen (eds.): Guiding the Invisible Hand. Economic Liberalism and the State in Latin American History, Praeger Publishers, New York, 1988.

25. Félix Luna, Soy Roca, Ed. Sudamericana, Buenos Aires, 1989. 26. Rosendo Fraga, El hijo de Roca, Emecé, Bs. As., 1994. 